\title{
Participation of Fishing Communities in Neglected Tropical Disease/HIV Co-infection Cohort Studies in Western Kenya
}

\author{
Erick MO Muok ${ }^{1^{*}, 2}$, Daniel O Onguru ${ }^{1,3}$, Diana M Karanja ${ }^{1}$, Pauline NM Mwinzi ${ }^{1}$, Zipporah W Nganga ${ }^{2}$ and Ayub V Ofula ${ }^{3}$ \\ ${ }^{1}$ Centre for Global Health Research, Kenya Medical Research Institute, Kisumu, Kenya \\ ${ }^{2}$ Institute of Tropical Medicine and Infectious Diseases/Jomo Kenyatta University of Agriculture and Technology, Nairobi, Kenya \\ ${ }^{3}$ Maseno University, Maseno, Kenya
}

\begin{abstract}
Background: Fishing communities on the shores of Lake Victoria are at high risk of schistosome infections, and at the same time, also considered at high risk of HIV infection due to factors associated with lifestyle or poor access to health services. In the absence of targeted interventions, the burden of schistosome-HIV co-infections continues to be high among such populations.

Methods: Fishing communities living along the shores of Lake Victoria were recruited into immunological cohort studies investigating the host-parasite interactions in HIV-Schistosome co-infections. We assessed the prevalence and incidence of both schistosome and HIV infections among fishing communities in western Kenya, and compared retention rates and ease of follow-up in two completed immunological cohort studies.

Results: At least 25 busy fishing sites were identified and six beaches were selected to participate in the studies. Up to 314 adults consented to participate in the immunological study on the role of human B cells in resistance to reinfection with S. mansoni (Cohort 1). Only 214 were able to provide baseline blood samples, while 97 and 34 were followed for the first and second times, respectively. Of the 191 tested for HIV, 62 (32.5\%) were HIV-positive. After one year HIV seroprevalence had risen to $36.1 \%$ among 108 participants who were screened. In the second cohort, 1040 potential participants were approached to participate. Up to 138 (13.3\%) did not return after VCT. A total of 622 persons who were HIV positive were enrolled. Overall $35.8 \%$ of those eligible for the study had HIV/schistosomiasis co-infection. The prevalence of other soil transmitted helminthes was: hookworms at $3 \%$, Ascaris lumbricoides at $<1 \%$ and Trichuris trichiura at about $1.5 \%$.

Conclusions: HIV remains an important factor to consider when planning for neglected tropical diseases (NTD) community based medical interventions in the region, and HIV interventions should also take into consideration the endemicity of prevalent NTDs in such settings. On the other hand, fishing communities could benefit from NTD/HIV co-infection study cohorts even though retention among these highly migrant communities is still a major issue to consider, while contributing to research development.
\end{abstract}

Keywords: HIV; Co-infection; Helminths; Western Kenya

\section{Introduction}

Historically, there has been a high prevalence of intestinal parasite infections (IPIs) among human populations in sub Saharan Africa and particularly in western Kenya, (Plate 1) [1]. Today, IPIs are still common in economically undeveloped rural areas in sub-Saharan Africa. A more recent survey around Kisumu city in close proximity to the study area indicated that $34 \%$ of sampled children were infected with one or more helminth species, whereas $16.2 \%$ of children were infected with one or more Soil Transmitted Helminths (STH) species. Schools in closest proximity to Lake Victoria and River Nyamasaria had the highest $S$. mansoni prevalence while schools with STH were more homogenously distributed. Mean school prevalence of S. mansoni infection was $21 \%$ while up to $20 \%$ had S. hematobium, hookworms, trichuriasis or ascariasis [1].

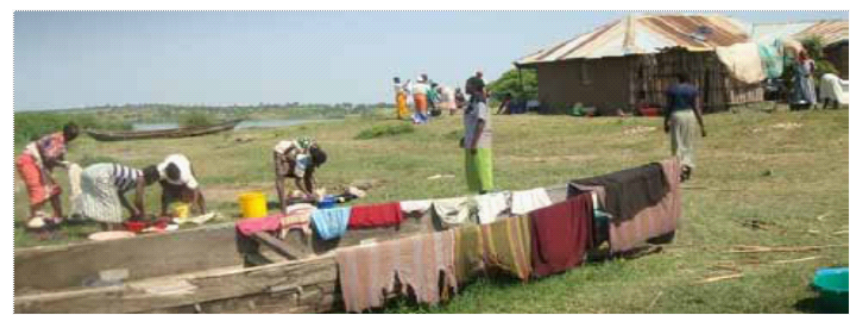

Plate 1: A fishing community on the shores of L. Victoria
S. mansoni infection is one of the most important and widespread of the neglected tropical diseases in western Kenya, especially among communities living around the shores of Lake Victoria [2,3]. With the spread of HIV in the resource constrained regions of the Lake shores where transmission is fuelled by lifestyle and poor access to health services, more and more people living with HIV are likely to be coinfected with other parasites [4]. The prevalence of HIV/schistosomiasis co-infection is very high in sub-Saharan Africa [5] and in particular in western Kenya [5], where schistosomiasis is also endemic.

Recent studies have shown that parasitic infections could disturb the balance of anti-HIV immune responses and contribute to HIV replication [6-8], which could accelerate progression to AIDS $[9,10]$. The reduced immune response caused by an HIV infection might

*Corresponding author: Erick MO Muok, Centre for Global Health Research, Kenya Medical Research Institute, Kisumu, Kenya, E-mail: Emuok@kemricdc.org

Received February 07, 20123; Accepted March 18, 2013; Published March 21 2012

Citation: Muok EMO, Onguru DO, Karanja DM, Mwinzi PNM, et al. (2013) Participation of Fishing Communities in Neglected Tropical Disease/HIV Coinfection Cohort Studies in Western Kenya. J Trop Dis 1: 104. doi:10.4172/2329-891X.1000104

Copyright: (c) 2013 Muok EMO, et al. This is an open-access article distributed under the terms of the Creative Commons Attribution License, which permits unrestricted use, distribution, and reproduction in any medium, provided the original author and source are credited. 
also lead to a higher susceptibility to parasitic infections. The high prevalence of certain opportunistic parasites among HIV positive persons is well known [11,12]. Such co-infections present with more severe clinical symptoms compared to parasite infections of otherwise healthy people, and are more difficult to treat [13].

Parasite - HIV co-infections is an area in HIV research that has not received much attention although HIV generally continues to be a major public health concern in the sub Saharan Africa and beyond. We assessed the prevalence of both schistosome and HIV infections among fishing communities in western Kenya, and compared retention rate and ease of follow-up in two ongoing immunological studies in order to inform designing of future studies and interventions aimed at prevention and control of co-infections including treatment needs of HIV/AIDS patients.

\section{Materials and Methods}

\section{Study area and population}

All participants were from fishing communities along the shores of Lake Victoria who were recruited into two immunological studies investigating host-parasite interactions in HIV-schistosome coinfections following informed consent. The six participating sites were chosen from a possible list of 25 busy fishing beaches lying between Kisian (Kisumu Municipality) and Luanda K’Otieno (Rarieda), along the lakeshores of Lake Victoria. More than half of the beaches were inaccessible by car, especially in Madiany Division (Uyoma). Madiany Division (Uyoma) is located within the shores of Lake Victoria in the Nyanza Province in western Kenya. The area is highly endemic for S. mansoni. A 2001 survey of schools in this area found S. mansoni prevalence ranging from $35-60 \%$ [3]. The six selected sites were identified after considerations based on logistical factors including access to fishing beach from the main road, population of fishermen and fish handlers, and presence of organized beach management unit (BMU).

\section{Survey processes}

The study of immunological investigations of the role of human $B$ cells was carried out between September 2008 and March 2010 while the study investigating immune reconstitution inflammatory syndrome (IRIS), which affects people receiving HIV treatment while harboring latent infections was carried out from May 2010 to September 2011. After 2 months of study introduction to the community, training of community representatives, field workers at each beach, health education and awareness campaigns for potential study participants, persons working in the beaches and willing to participate in the studies were consented and enrolled in the surveys. Each participant was assigned a unique identifier and referred to the Kenyan Ministry of Health owned/run VCT centers for HIV testing. Participants who presented a VCT card (without results) as evidence of having received counseling and testing for HIV were then asked to provide a stool sample for examination of $S$. mansoni eggs and other intestinal worms.

\section{Parasitological testing}

Standard Kato/Katz thick smears method was used for stool examinations for schistosomiasis and other helminth eggs [14], using 2 slides per stool. These slides were then quantitatively evaluated by well trained laboratory technicians. Quality control was assured by randomized selection and examination of $25 \%$ of the samples by another technician who was blinded for the first results. This technique was used to diagnose infections with S. mansoni, Ascaris lumbricoides, hookworm, and Trichuris trichiura. Urine was tested for presence of blood using Ames Hemastix reagent strips (Miles, Inc., Elkart, IN) as a marker of possible S. haematobium infection.

\section{Ethical considerations}

The two study protocols were approved by the two tier scientific and ethical institutional review committees of the Kenya Medical Research Institute in Nairobi, Kenya. Participants were contacted through trained community representatives at the beaches. The study objectives, procedures, potential risks and benefits were carefully explained to all potential participants by study staff. Interested individuals provided written informed consent before inclusion in the study.

All participants were offered professional counseling before and after HIV testing by staff from the local HIV/AIDS clinics (managed by the Kenyan Ministry of Health), and all diagnostic results were kept strictly confidential. Deworming drugs (albendazole, praziquantel) were administered to all participants found to be infected with helminths by the study nurse.

\section{Results}

\section{Study cohort 1}

Studies investigating the role of human B cells in resistance to reinfection with S. mansoni: Following study introduction and community training, 314 adults (male fishermen) consented to participate in the immunological study on the role of human B cells in resistance to reinfection with $S$. mansoni. Only 214 (68\%) were able to provide baseline blood samples, while 97 and 34 were followed through the second and third year, respectively. Since this cohort 1 (Table 1 and figure 2) consisted exclusively of fishermen, there was a very high frequency of movement in search of fish resulting in $74.4 \%$ loss to follow-up, other losses to follow-up were due to refusals accounting for $12.8 \%$ while the rest (12.2\%) were as a result of other reasons like death $(n=4)$, imprisonment $(n=2)$ or being bedridden with illness $(\mathrm{n}=5)$. Of the 191 tested for HIV at the start of the study, $62(32.5 \%)$ were HIV-positive. After one year, HIV sero-prevalence had risen to $36.1 \%$ following screening of 108 participants. The heavy losses up to $50 \%$ especially to movement in search of fish would have an influence on the data since it can possibly be viewed as those already having HIV were likely to be left behind due to their inability to perform fully their duties.

\section{Study cohort 2}

Studies investigating Immune Reconstitution Inflammatory Syndrome (IRIS): One thousand and forty (1040) potential

\begin{tabular}{|l|c|c|c|c|}
\hline & N & Mean & Median & Range \\
\hline Participant Age & 257 & 34.82 & 31 & $17-75$ \\
\hline Years resident in the area & 110 & 22.14 & 20 & $1-66$ \\
\hline Years worked as fisherman & 110 & 11.8 & 9.5 & $1-45$ \\
\hline
\end{tabular}

Table 1: Demographic characteristics of study populations recruited in the first cohort.

\begin{tabular}{|l|l|l|l|}
\hline & $\begin{array}{l}\text { S. mansoni egg } \\
\text { positive (+ve)at } \\
\text { baseline (n=53) }\end{array}$ & $\begin{array}{l}\text { S. mansoni egg } \\
\text { negative (-ve) at } \\
\text { baseline (n=46) }\end{array}$ & p-value \\
\hline Age in years [mean (std. dev)] & $36.8(10.1)$ & $35(9.1)$ & 0.3646 \\
\hline Years around the lake [mean (std)] & $21(2.2)$ & $23(3.1)$ & 0.5661 \\
\hline Gender [male (n) (\%)] & $31(54.39)$ & $13(37.1)$ & 0.1013 \\
\hline
\end{tabular}

Table 2: Demographic characteristics of study populations recruited in the second cohort. 
participants were approached to participate in the cohort 2 (Table 2) study investigating immune reconstitution inflammatory syndrome. IRIS affects people receiving HIV treatment while harboring latent infections. Up to 280 (26.9\%) of them tested HIV negative and therefore were not eligible for enrollment into the study, while $64.3 \%$ of the HIV negative participants were S. mansoni positive. Overall 59.8\% had HIV/ schistosomiasis co-infection (622 out of 1040). Other soil transmitted helminthes were also recorded with the following prevalence: hookworms at 3\% overall, Ascaris lumbricoides at $<1 \%$ and Trichuris trichiura at about 1.5\% (Figure 1). Comparatively, Cohort 2 had a better follow-up retention possibly because all eligible adults regardless of gender were included in the study. Women were more likely to remain in the study area compared to men as observed in the second cohort where 52 women were able to complete at least three months follow up compared to 40 males. Cohort 1 was used to inform cohort 2 and lessons learnt in the previous cohort were crucial in the implementation of the second study cohort.

The study investigating the role of human B cells in resistance to re-infection with $S$. mansoni showed that CD23 may be involved in the development of resistance to schistosome infection through its role in IgE regulation. The immune reconstitution inflammatory syndrome (IRIS) study results showed that Schistosoma-associated IRIS in patients with HIV/S. mansoni co-infection develops generally within three months upon successful HAART. Case definition specific to Schistosoma-associated IRIS involves radiological examination showing worsening or emergence of hepato-splenomegaly and vein enlargement, increase in CD4+ lymphocyte count and significant reduction in viral load count with adequate adherence to ART (Unpublished data), this definition will be useful to clinicians and researchers in schistosomiasis endemic settings for identification and management of Schistosomaassociated IRIS.

\section{Discussions}

We describe fishing community sites that present priority populations for intervention and investigations related to NTD/
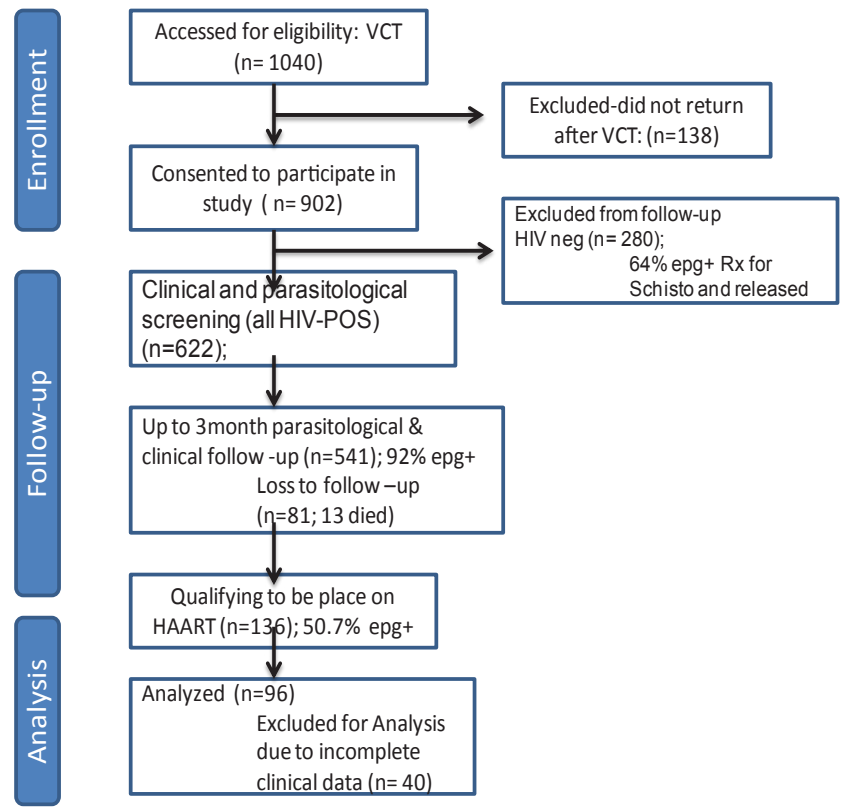

Figure 1: Schematic representation of Cohort 2.

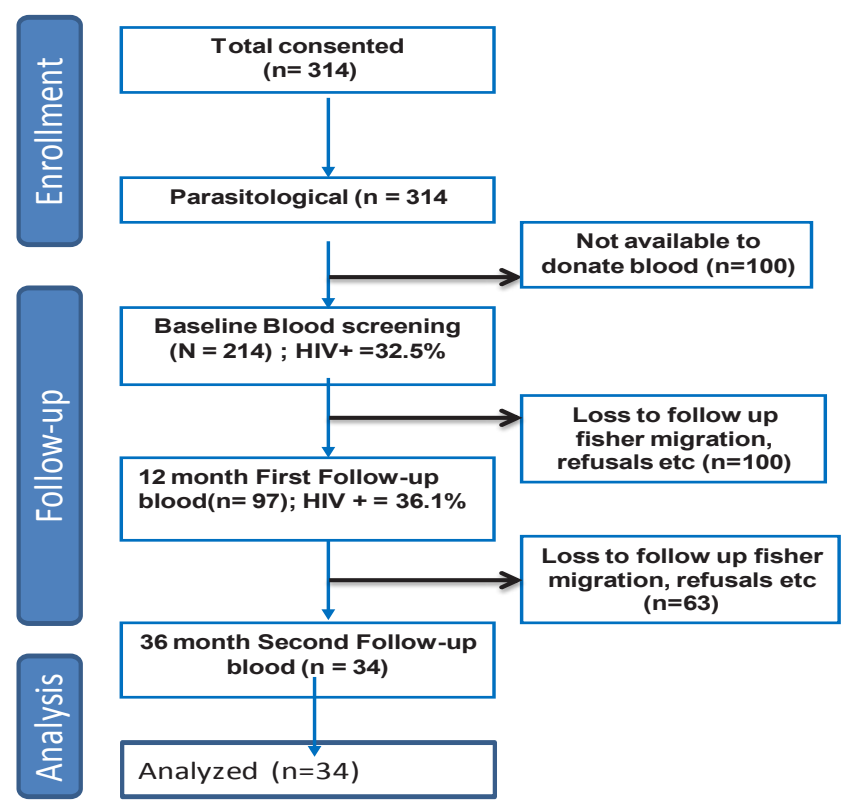

Figure 2: Schematic representation of Cohort 1.

HIV co-infections due to high incidence rates of HIV and prevalent NTDs. Prioritizing such sites for research studies will also ensure access to urgently needed interventions in such settings. At the same time, disease management strategies and timing of treatments for coinfected individuals are areas where urgent data is needed to inform policy. We recorded very high dropout rates with up to less than half of the fishermen being followed up two times in cohort 1 with the main contributor being refusals and movement in search for fish among others like death, imprisonment and serious illnesses.

Involvement of fishermen in study participation led to the highest study drop out rate but still an adequate number managed to complete the study especially in the second cohort that got informed by the experiences of the first. This is consistent with literature that records vulnerability of fishing communities to HIV/AIDS and other diseases has been widely overlooked. The consequence is that they have been left largely beyond the reach of prevention, care and mitigation efforts. This neglect is having devastating consequences. In the last decade, it has become evident that fishermen in many developing country fishing communities suffer from HIV prevalence rates often five to ten times higher than those in the general population.

Interventions targeting fishermen should therefore build in mechanisms for tracing and follow up, to monitor their migration patterns and to consider such patterns into intervention designs. The data suggests considerable number of deaths that could be attributed to low adherence to HIV treatment as a result of movements. Among the study participants included in the second cohort, there was high prevalence of $S$. mansoni in HIV negative individuals as well as HIV/ schistosomiasis co-infection. Other soil transmitted helminthes were also recorded to be lower than the national survey results with the highest in terms of prevalence being Hookworms followed by Ascaris and Trichuris trichiura. This second cohort had a better retention possibly because all adults were inclusive and since women were occupationally not likely to by migrate by virtue of being more involved in house duties, they are also more likely not to move away from field sites and therefore had a better follow-up record compared to men. 
Citation: Muok EMO, Onguru DO, Karanja DM, Mwinzi PNM, et al. (2013) Participation of Fishing Communities in Neglected Tropical Disease/HIV Co-infection Cohort Studies in Western Kenya. J Trop Dis 1: 104. doi:10.4172/2329-891X.1000104

The second cohort also most probably benefited from lessons related to retention from the previous cohort and that possibly played a very crucial part in the success of the second cohort. The helminthic prevalence values are considerably lower than those reported from the nationwide survey [15] except for $S$. mansoni which could be due to differences in transmission. The nationwide prevalence survey of these infections in this area reported $28.6 \%$ overall with A. lumbricoides at $13.3 \%$. Hookworm $s p$. at $55.7 \%$ and T. trichiura at $4.9 \%$ showed decline in helminthic prevalences which can be attributable to the economical development of the country resulting in increased urbanization, infrastructure development and increased health consciousness.

The fishing communities due to their geographic isolation, low literacy levels, high mobility, general attitudes towards risk, and the near absence of a wide range of basic health services, makes them vulnerable to a variety of infectious diseases as our survey indicates and as might be expected, they have been hit especially hard by the HIV epidemic. Early data emerging from an ongoing study conducted by the Uganda Virus Research Institute (UVRI) and funded by the European and Developing Countries Clinical Trials Partnership (EDCTP) revealed that $27.5 \%$ of their fishing population is living with HIV, compared to a national average of $6.4 \%$ [16].

This is comparable with our data in both cohorts having HIV prevalence at $35.7 \%$ and $32.5 \%$ respectively for cohort one and two against a national average of $6.9 \%$ according to UNAIDS and the WHO's report on the Global health Epidemic [17]. Overall it has been estimated that more than a third of all individuals in sub-Saharan Africa are infected with at least one species of helminth, with considerable overlap in the prevalence of helminthes and HIV-1 [18,19]. According to our data this is higher in the fishing communities making them priority targets for more intervention strategies that are suitable for such unstable populations to be coupled with the clinical evaluations. Thus, co-infection with HIV-1 and helminths is common among adults in Kenya and more particularly among the fishing communities which includes more than one million people living in the along the shores and islands of Lake Victoria. We therefore present unique cohorts in which marginalized communities may benefit from participation in basic and operational research aimed at HIV and NTD prevention and control, and the acceptance of such research. This information should be useful in designing interventions in these settings.

\section{Acknowledgements}

The authors would like to thank Mr. Boaz Mulonga, Martin Ogemba, Samwe Andiwo, Fridah Mualma, George Ogolla, field workers and especially all the study participants. This study is published with the permission of the Director, Kenya Medical Research Institute.

\section{References}

1. Odiere MR, Opisa S, Odhiambo G, Jura WG, Ayisi JM, et al. (2011) Geographical distribution of schistosomiasis and soil-transmitted helminths among school children in informal settlements in Kisumu City, Western Kenya. Parasitology 138: 1569-1577.

2. Black CL, Mwinzi PN, Muok EM, Abudho B, Fitzsimmons CM, et al. (2010) Influence of exposure history on the immunology and development of resistance to human Schistosomiasis mansoni. PLoS Negl Trop Dis 4: e637.

3. Handzel T, Karanja DM, Addiss DG, Hightower AW, Rosen DH, et al. (2003) Geographic distribution of schistosomiasis and soil-transmitted helminths in Western Kenya: implications for anthelminthic mass treatment. Am J Trop Med Hyg 69: 318-323.
4. Shao Y (2006) AIDS epidemic at age 25 and control efforts in China Retrovirology 3: 87.

5. Mwinzi PN, Karanja DM, Kareko I, Magak PW, Orago AS, et al. (2004) Short report: Evaluation of hepatic fibrosis in persons co-infected with Schistosoma mansoni and human immunodeficiency virus 1. Am J Trop Med Hyg 71: 783786

6. Newton CR (2005) Interaction between Plasmodium falciparum and human immunodeficiency virus type 1 on the central nervous system of African children. J Neurovirol 11: 45-51.

7. Gopinath R, Ostrowski M, Justement SJ, Fauci AS, Nutman TB (2000) Filaria infections increase susceptibility to human immunodeficiency virus infection in peripheral blood mononuclear cells in vitro. J Infect Dis 182: 1804-1808.

8. Harms G, Feldmeier H (2002) HIV infection and tropical parasitic diseases deleterious interactions in both directions? Trop Med Int Health 7: 479-488.

9. Karanja DM, Colley DG, Nahlen BL, Ouma JH, Secor WE (1997) Studies on schistosomiasis in western Kenya: I. Evidence for immune-facilitated excretion of schistosome eggs from patients with Schistosoma mansoni and human immunodeficiency virus coinfections. Am J Trop Med Hyg 56: 515-521.

10. Tawill SA, Gallin M, Erttmann KD, Kipp W, Bamuhiiga J, et al. (1996) Impaired antibody responses and loss of reactivity to Onchocerca volvulus antigens by HIV-seropositive onchocerciasis patients. Trans R Soc Trop Med Hyg 90: 8589.

11. Karp CL, Auwaerter PG (2007) Coinfection with HIV and tropical infectious diseases. II. Helminthic, fungal, bacterial, and viral pathogens. Clin Infect Dis 45: $1214-1220$.

12. Nielsen NO, Simonsen PE, Dalgaard P, Krarup H, Magnussen $P$, et al. (2007) Effect of diethylcarbamazine on HIV load, CD4\%, and CD4/CD8 ratio in HIVinfected adult Tanzanians with or without lymphatic filariasis: randomized double-blind and placebo-controlled cross-over trial. Am J Trop Med Hyg 77: 507-513.

13. Corbett EL, Steketee RW, ter Kuile FO, Latif AS, Kamali A, et al. (2002) HIV-1/ AIDS and the control of other infectious diseases in Africa. Lancet 359: 2177 2187.

14. Katz N, Chaves A, Pellegrino J (1972) A simple device for quantitative stool thick-smear technique in Schistosomiasis mansoni. Rev Inst Med Trop Sao Paulo 14: 397-400

15. Walson JL, Stewart BT, Sangaré L, Mbogo LW, Otieno PA, et al. (2010) Prevalence and correlates of helminth co-infection in Kenyan HIV-1 infected adults. PLoS Negl Trop Dis 4: e644.

16. Kidega W (2010) Casting a finer net: involving fishing communities in HIV research. Health Exchange.

17. UNAIDS Report on the Global AIDS Epidemic - 2010, HIV estimates with uncertainty bounds.

18. Bethony J, Brooker S, Albonico M, Geiger SM, Loukas A, et al. (2006) Soiltransmitted helminth infections: ascariasis, trichuriasis, and hookworm. Lancet 367: 1521-1532.

19. Borkow G, Bentwich Z (2004) Chronic immune activation associated with chronic helminthic and human immunodeficiency virus infections: role of hyporesponsiveness and anergy. Clin Microbiol Rev 17: 1012-1030. 